\title{
ITGB6 promotes the proliferation and invasion in pancreatic cancer
}

\section{Type}

Research paper

\section{Keywords}

pancreatic cancer, biomarker, cancer progression, ITGB6, Functional assay

\begin{abstract}
Introduction

ITGB6 gene encoding a protein that can regulate the integrin av $\beta 6$ heterodimer protein expression in different status was showed that it played an important role in multiple human cancers, such as brain cancer, colon cancer and oral cancer, and related to the clinical progression. This study is to explore the function and the mechanism of ITGB6 gene or protein in pancreatic cancer.
\end{abstract}

Material and methods

We examined the expression of ITGB6 in pancreatic cancer using immunohistochemistry and analyzed the correlationship between the expression of ITGB6 and the clinicalpathologic features in pancreatic cancer patients. In addition, bioninformatic method was conducted to analyze the ITGB6 mRNA level in pancreatic tumor tissues compared with normal pancreatic tissues and to analyze the correlationship between high KIF23 expression and prognosis in pancreatic cancer patients.

Moreover, colony formation assay, MTT assay, cell scratch, cell invasion and western blot assays in vitro and xenograph mice model in vivo were performed to analyze the effect of KIF23 on proliferation and invasion of pancreatic cancer cells.

Results

We reported that increased expression of ITGB6 was significantly correlated with poor clinical outcome in both our clinical data and TCGA data of pancreatic cancer. Furthermore, functional assays revealed that knockdown ITGB6 in vivo and in vitro might inhibit cancer cells proliferation and the ability of invasion or migration.

\section{Conclusions}

Our data suggests that ITGB6 is associated with pancreatic cancer malignant progression. Hence, ITGB6 may be served as a potential target of pancreatic cancer in clinical treatmentfor future research, and further study is needed. 


\section{ITGB6 may promote the proliferation and invasion in pancreatic cancer}

Chao Zhong (ChaoZhong1981@ 163.com) ${ }^{1 \#, ~ Z h i-X i ~ L i ~(L i z h i x i 246 @ ~ s i n a . c o m) 2 \#, ~ L i n g-J i n g ~}$ Yang (Yanglingjing1129@163.com) ${ }^{2}$, Gang Wu (wugang@ med.uestc.edu.cn) ${ }^{3}$, Bo Xiang (Alexxiang44@163.com) ${ }^{4}$, Yu-Lan Wang (158728570@qq.com) $)^{5}$, Qing Zhou (uszhouqing@163.com) ${ }^{6 *}$

${ }^{1}$ Department of traditional chinese medicine of orthopedic and traumatic, ${ }^{2}$ Department of Respiratory Medicine, East hospital, ${ }^{3}$ Department of Hepatobiliary Surgery, ${ }^{4}$ Department of cardiosurgery, ${ }^{5}$ Department of oncology, ${ }^{6}$ Department of ultasound, Academy of Medical Sciences \& Sichuan Provincial People's Hospital, No.32 west second section First Ring Road, Chengdu city, 610072, Sichuan province, China. 86-02867897856.

\# The authors contribute equally to this study. *Corresponding author.

Runing title: ITGB6 may be associated with pancreatic cancer malignant progression.

Abstract:

Background and aims ITGB6 gene encoding a protein that can regulate the integrin $\alpha v \beta 6$ heterodimer protein expression in different status was showed that it played an important role in multiple human cancers, such as brain cancer, colon cancer and oral cancer, and related to the clinical progression. This study is to explore the function and the mechanism of ITGB6 gene or protein in pancreatic cancer. Methods We examined the expression of ITGB6 in pancreatic cancer using immunohistochemistry and analyzed the correlationship between the expression of ITGB6 and the clinicalpathologic features in pancreatic cancer patients. In addition, bioninformatic method was conducted to analyze the ITGB6 mRNA level in pancreatic tumor tissues compared with normal pancreatic tissues and to analyze the correlationship between high KIF23 expression and prognosis in pancreatic cancer patients. Moreover, colony formation assay, MTT assay, cell scratch, cell invasion and western blot assays in vitro and xenograph mice model in vivo were performed to analyze the effect of KIF23 on proliferation and invasion of pancreatic cancer cells. Results We reported that increased expression of ITGB6 was significantly correlated with poor clinical outcome in both our clinical data and TCGA data of pancreatic cancer. Furthermore, functional assays revealed that knockdown ITGB6 in vivo and in vitro might inhibit cancer cells proliferation and the ability of invasion or migration. Conclusions Our data suggests that ITGB6 is associated with pancreatic cancer malignant progression. Hence, ITGB6 may be served as a potential target of pancreatic cancer for future research, and further study is needed.

Keywords: Pancreatic cancer, Cancer progression, ITGB6, Functional assay, Biomarker. 


\section{Article summary}

Strengths and limitations of this study: We first reported that increased expression of ITGB6 was significantly correlated with poor clinical outcome in both our clinical data and TCGA data of pancreatic cancer. Furthermore, functional assays revealed that knockdown ITGB6 in vivo and in vitro might inhibit cancer cells proliferation and the ability of invasion or migration. Our data suggests that ITGB6 is associated with pancreatic cancer malignant progression. Hence, ITGB6 may be served as a potential target of pancreatic cancer in clinical treatment. However, the results should be proved by other studies with more patients in the multi-center studies, and the mechanism of ITGB6 in pancreatic cancer should be further explored.

\section{Introduction:}

Pancreatic cancer as a highly malignant tumor is predicted to be the second cause of cancerassociated death in the next decade[1,2]. Rapid spreading in patients and difficulty to detect in the early stages made pancreatic cancer become the leading cause of cancer-related death[3]. The cancer mortality is extremely high, though the substantial improvement had achieved in the fields of early diagnosis, surgical techniques, combination chemotherapies and other anti-cancer treatments for patients with pancreatic cancer[4,5]. In common, there are no apparent symptoms or signs in patients unless this malignancy progresses into an advanced stage[6]. Once in advanced stages, the chance of surgery has been lost. Therefore, it's of vital importance that clinical physicians should identify the patients with high risk. The cancer specific 5-year overall survival is less than 5\%[7]. Moreover, patients proceeded curative surgical resection still relapse in the following 2 years. Hence, the mechanisms of cancer development and progression were needed to be unveiled and for cancer treatment, potential biomarkers and novel anti-cancer strategies are urged to be identified[8].

Integrins, as adhesion receptors that play an important role in signaling from the extracellular matrix to the cell, are composed of an alpha chain and a beta chain[9,10]. Integrins mediate critical cell signaling through as key elements of conducting inside-out and outside-in signaling. For heterodimeric trans-membrane proteins, the subunits are capable of forming different heterodimers[11,12]. The integrin subunit beta 6 (ITGB6) gene encodes a protein that belongs to integrin superfamily and as a vital subunit of the integrin $\alpha v \beta 6$ heterodimer can regulate this heterodimer protein expression level in different status[13,14]. The integrin $\alpha v \beta 6$ as receptors of ECM proteins and TGF- $\beta$ 's latency-associated peptide regulates key biological process, including fetal development and wound healing[15-17]. Previous studies also reported that it might involve in pathological processes and carcinogenesis[18,19]. The integrin $\alpha v \beta 6$ constitutively expresses in healthy epithelia, normally, while during carcinogenesis the expression level up-regulated[20]. Moreover, the ITGB6 mainly regulates the av $\beta 6$ expression owing to its important role in forming heterodimer with $\alpha \mathrm{v}$ proteins. ITGB6 is largely expressed in developing tissues, wound healing process and human epithelial malignancies but not in the normal epithelia. Recent studies showed 
that ITGB6 plays an important role in multiple human cancers, such as brain cancer, colon cancer and oral cancer, and related to the clinical progression[21-23]. Hence, to uncover the ITGB6 role in pancreatic cancer is of vital importance.

In the present study, we demonstrated that high ITGB6 expression was significantly correlated with poor clinical outcomes in pancreatic cancer patients and further functional assays revealed that knockdown ITGB6 in vivo and in vitro might inhibit cancer cells proliferation and the ability of invasion or migration. Owing to the data we presented, ITGB6 may be a prognostic biomarker for pancreatic cancer and served as a novel target in the clinical treatment.

\section{Materials and methods}

In this section, we followed the methods of Zheng et al. 2015 and Niu et al. 2017 [24,25].

\section{Patient and Public Involvement}

This retrospective study identified 82 consecutive patients with PDAC who underwent pancreatic cancer resection in our hospital from May 2012 to May 2017. Patients were classified according to the 2009 UICC TNM staging and the 2004 WHO/ISUP classification. All patients had no preoperative radiotherapy and/or chemotherapy and neoadjuvant chemotherapy. Attendees provided written informed consent. All patients showed no evidence of tumor metastasis confirmed by cross-sectional imaging.

\section{Immunohistochemistry}

Pancreatic cancer specimens were stained for detection the ITGB6 expression. Pancreatic cancer tissues were cut in $3.5 \mu \mathrm{m}$ thick and pretreated in Tris-buffered saline. After antigen retrieval, samples were incubated with primary antibody rabbit anti-ITGB6 (1:200 dilution, ab233519, Abcam, Cambridge, UK) at $4^{\circ} \mathrm{C}$ overnight. Next, sections were washed twice with Tris-buffered saline and incubated for $30 \mathrm{~min}$ at room temperature with the blocking buffer of 5\% conjugated secondary antibody. Subsequently, diaminobenzidine (DAB) were dripped on in 3-5 minutes. The images were collected by microscope (Nikon, Tokyo,Japan).

ITGB6 protein is mainly located in the cell membrane of tumor cells. The tissues were scored according to the proportion of positive tumor cells and the staining intensity of tumor cells. The proportion of positive tumor cells $<10 \%$ score 0 ; The percentage of positive tumor cells occupies $10 \%$ to $30 \%$ score 1 . The positive percentage of tumor cells was $30 \%-70 \%$ score 2 , and the positive percentage of tumor cells was $70 \%>$, score 3 . The positive staining was evaluated zero for negative staining, 1 for weak positive staining, 2 for moderate positive staining, and 3 for strong positive staining. The final scores were calculated by multiplying the proportion and intensity. Results high expression (>3) or low expression (0-3) were determined according to the score of positive cell percentage score and staining intensity score. The sections of each patient were observed within 3 visual fields, and two experienced pathologists read the sections without 
getting the pathological grade and clinical data. The results were judged by double-blind method.

\section{Cell culture and reagents}

Human pancreatic cancer cell lines PANC-1 and BxPC-3 were purchased from China Center for Type Culture Collection. The above cells were cultured in high-glucose Dulbecco's modified Eagle's medium (DMEM) and RPMI, respectively, all supplemented with $10 \%$ fetal bovine serum (FBS), $100 \mathrm{U} / \mathrm{ml}$ penicillin, and $0.1 \mathrm{mg} / \mathrm{ml}$ streptomycin. All cells were maintained at $37^{\circ} \mathrm{C}$ in a $5 \% \mathrm{CO}_{2}$ humidified incubator.

\section{Stable cell line generation}

The human ITGB6 (gene ID: 80381) targeting small hairpin (sh) RNA was purchased from Genechem (Lot: TR312078, Genechem Co., Ltd, Shanghai, China) and non-effective 29-mer scrambled shRNA (Lot: TR30012; Genechem Co., Ltd, Shanghai, China). Recombinant lentivirus was tittered to $2 \times 10^{8} \mathrm{TU} / \mathrm{mL}$, and interested cells were transfected by the lentivirus with the 20 of multiplicity of infection (MOI). RT-qPCR and Western blotting were performed to confirm the effect of ITGB6 silencing.

\section{RNA analysis}

Total RNA was extracted using TRIzol reagent (Invitrogen) and confirmed the RNA integrity. PrimeScript RT reagent kit (Invitrogen) were used for cDNA generation. RT-qPCR was performed with SuperReal PreMix (Tiangen Biotech) by the following program: $95^{\circ} \mathrm{C}$ for $3 \mathrm{~min}$ in 1 cycle; $95^{\circ} \mathrm{C}$ for $5 \mathrm{~s}, 58^{\circ} \mathrm{C}$ for $30 \mathrm{~s}$, and $72^{\circ} \mathrm{C}$ for $30 \mathrm{~s}$ in 35 cycles. To ensure the DNA production, a melting curve analysis was performed according to ABI StepOne system. The relative gene expression was normalized to the internal standard $\beta$-actin using $2-\Delta \Delta \mathrm{Ct}$ method. All experiment was performed independently for three times. Primers used for the analysis are as follow:

ITGB6 F: 5'-CTTTCCCTAGCCTGCCTTCT-3',

ITGB6 R: 5'-GTTCAATCCCCATCCGTTT-3';

$\beta$-actin F: 5'- CAGCTCACCATGGATGATGATATC -3',

$\beta$-actin R: 5'- AAGCCGGCCTTGCACAT -3'.

\section{Immunoblotting}

Cells were lysed in lysis buffer $(60 \mathrm{mM}$ Tris-HCl, pH6.8, 2\% SDS, 20\% glycerol, $0.25 \%$ bromophenol blue, 1.25\%2-mercaptoethanol and protease inhibitor cocktail). Lysates were used the BCA Protein Assay (Thermo Fisher Scientific) to measure concentration followed by manufacturer's instructions. Next, the equal amounts of protein $(30-50 \mathrm{mg})$ was electrophoresed by $10 \%$ SDS-PAGE gels and transferred onto PVDF membranes (Merck Millipore). Then, using 
non-fat milk to block the membrane, the membranes were incubated with antibodies at $4^{\circ} \mathrm{C}$ overnight. The antibodies used are showed as follow: rabbit anti-ITGB6 (1:1000 dilution, ab233519, Abcam, Cambridge, UK); mouse anti- $\beta$-actin (1:1000 dilution, ab8226, Abcam, UK), rabbit anti-Ki67 (1:1000 dilution, ab16667, Abcam, UK), mouse anti-proliferating cell nuclear antigen (PCNA) (1:500 dilution, ab29, Abcam, UK), mouse anti-MMP2 (1:1000 dilution, ab37150, Abcam, UK) and mouse anti-MMP9 (1:1000 dilution, ab38898, Abcam, UK).

\section{Colony formation assays}

Cell proliferation ability was performed by colony formation assay. 400 cells per well were plated in 6-well plates. Cells were fixed in methyl hydrate for 10 minutes, accordingly, and the colonies were stained and counted by using an optical microscope.

\section{MTT assay}

MTT assay was used to study cell proliferation ability. $3 \times 10^{3}$ cells were plated in a 96-well plate, and $10 \mathrm{ul}$ of $5 \mathrm{mg} / \mathrm{ml}$ MTT was added into each well and continued to culture for 4 hours. Adding dimethyl sulfoxide into the plate, then the plate was placed on a microplate autoreader (Bio-Rad, Hercules) and acquire optical density at $570 \mathrm{~nm}$ wavelength. Cell growth curves were generated to determine the optical density value.

\section{Cell scratch assays}

The interested cells were seeded in 6-well plates and grew into full confluence overnight. A sterile pipette tip was used to introduce a scratch in the middle of each well. Next, the growth medium was discarded and replaced with fresh medium. The rate of migration towards the center of the wound was ascertained at indicated time points.

\section{Cell invasion assays}

In migration or invasion assay, $3 \times 10^{5}$ cells were plated in the upper chambers coated with Matrigel (BD Bioscience, Mountain View, CA, USA). The following growth medium were added into the lower chamber and cultured at $37^{\circ} \mathrm{C}$ for 24 hours. Subsequently, cells migrated to the lower side of membrane were fixed and stained. The cells were calculated at three randomly selected fields by microscope from each chamber.

\section{Growth assay in vivo}

The in vivo animal study was approved by Tianjin Medical University Cancer Institute and Hospital. The immunocompromised nude mice (aged 4-5 weeks) were purchased from Beijing Vital River Laboratory Animal Technology Co., Ltd. (Beijing, China). The $1 \times 10^{6}$ non-effected shRNA treated cells and $1 \times 10^{6}$ ITGB6 shRNA treated cells were injected subcutaneously into the mice. After 4 weeks inoculation, the final volume of tumor was weighed.

\section{Statistics}


All data were analyzed with SPSS 22.0 software. Connection between ITGB6 expression level and patients' survival time after surgery were performed by Kaplan-Meier method. Statistical comparisons were conducted by Student's t-test. The categorical data were analyzed by $\square \square$ method. The value of statistical significant was considered as $P<0.05$.

\section{Results}

Increased expression of ITGB6 connected with poor clinical outcomes in pancreatic cancer patients

In our clinical data of 78 pancreatic adenocarcinoma patients, we divided them into two groups: the ITGB6 low group $(n=28)$ and ITGB6 high group $(n=50)$ based on the protein expression level of ITGB6 (Table 1). Immunohistochemistry were used for detection the ITGB6 expression with low and high intensity in stained pancreatic cancer specimens (Fig 1A). Particularly, patients with high ITGB6 protein expression level were strongly connected to vicious TNM stage $(P=0.040)$, big tumor size $(P=0.039)$ and vascular invasion $(P=0.030)$ when compared to the low expression group. No significant clinical connection was determined between ITGB6 expression and other clinicopathological features, including age, gender, tumor grade, Lymph node metastasis (Table 1). To better investigate the ITGB6 clinical feature, Kaplan-Meier survival analysis was performed to determine the cancer overall survival (OS) in our 30 months follow-up time after surgery. The data showed that high ITGB6 protein expression level was related to a short cancer OS time and disease free survival time (Both $P<0.05$, Fig 1B).

To determine the ITGB6 mRNA expression level, bioinformation analysis was performed in an interactive web server GEPIA with the sequencing expression data of 9736 tumors. MRNA expression level of ITGB6 in PAAD (pancreatic adenocarcinoma) shows significantly higher than normal tissues $(P<0.05$, PAAD number=179, normal tissues number=171, Fig 2A). Further, we also investigated the cancer specific survival in PAAD dataset in GEPIA and showed that high ITGB6 expression was significantly connected with shorter overall survival and disease-free survival in PAAD patients ( $P=0.025$ and $P=0.041$, Fig $2 \mathrm{~B}$ and $2 \mathrm{C}$ ).

\section{Construction of lentivirus-based ITGB6 stable knockdown cell lines of PANC-1 and BxPC-3}

PANC-1 and BxPC-3 cells were transfected with recombinant lentivirus to silence of ITGB6 expression in cancer cells. Through RT-qPCR and Western blotting validation, we generated stable cell lines with both ITGB6 mRNA and protein level decreased significantly when compared with control cells (Fig 3). Using this model, we could efficiently study the ITGB6 role in cancer cells.

\section{Knockdown ITGB6 reducing proliferation through regulating Ki67 and PCNA proteins in of PANC-1 and BxPC-3 cell lines}

To investigate the biological effect of ITGB6, we conducted colony formation assay and MTT 
assay to evaluate the possible role for proliferation in pancreatic cancer cell lines PANC-1 and BxPC-3. Compared to control cells, stable knockdown ITGB6 in PANC-1 and BxPC-3 cells by recombinant lentivirus significantly decreased the ability of proliferation in vitro compare to control cells $(P<0.05$, Fig 4A and 4B). The above results may imply that knockdown ITGB6 reduced proliferation ability in cancer cells. To further investigate the mechanism of partially eliminated proliferation, we checked proliferation related proteins such as Ki67 and PCNA in vitro. Western blotting revealed that Ki67 and PCNA protein were significantly down-regulated in the silencing ITGB6 cell lines compared with the control, respectively $(P<0.05$, Fig 4C and 4D). Taken together, the above data suggested that knockdown ITGB6 may down-regulate Ki67 and PCNA protein to reduce the proliferation in pancreatic cancer cells.

\section{Through down-regulated MMP2 and MMP9 protein, ITGB6 silence inhibited migration or invasion in vitro.}

Cell scratch assay was used to study the cell migration changes. Comparing with non-effective scrambled shRNA treated cells, the ITGB6 silence cells showed bigger scratch space in PANC-1 and BxPC-3 cell lines, respectively (both $P<0.05$, Fig 5A). To investigate the possible role of regulating the cancer cells invasive capacity in stable knockdown ITGB6 cells, cell invasion assays were performed. It's obvious that the invasive capacity of ITGB6 shRNA treated cells tended to be partially lost compared to control cells after 48 hours (both $P<0.05$, Figure 5B). Moreover, to research the possible mechanism of the above changes, we checked invasion and metastatic associated proteins including matrix metalloproteinase (MMP)-2 and MMP9 at protein level by immunoblotting. The results showed that MMP2 and MMP9 in ITGB6 shRNA treated cells were lower than control (both $P<0.05$, Fig $5 \mathrm{C}$ and 5D). In a word, the above data suggested that through regulating the associated proteins, knockdown ITGB6 might inhibit the cancer cells' invasive capacity.

\section{In vivo inhibitory role of knockdown ITGB6}

Based the following results above, down-regulated ITGB6 in pancreatic cancer cell lines significantly inhibited cell proliferation and the ability of invasion or migration in vitro. To better investigate the above findings in vivo, xenograft assay was performed. The tumor volume of down-regulated ITGB6 group was observably smaller than control after 4 weeks of treatment $(P<0.05$, Fig 6A). In addition to further certify the effect of ITGB6 silencing, western blotting assay was performed to consolidate the ITGB6 silencing effect in mice tumors. The following data showed that ITGB6 was dramatically reduced by our recombinant lentivirus $(P<0.05$, Fig 6B). Taking together, our results suggested that knockdown ITGB6 may exert inhibitory effect in vivo.

\section{Discussion}


Pancreatic cancer is predicted to be the second cause of cancer-associated death in the next decade[1,2]. Recent studies have suggested that $\alpha \mathrm{v} \beta 6$ was overexpressed in various types of tumor and showed be closely bound up with invasive and metastatic features of cancer[23, 26, 27]. It's suggested that ITGB6 mainly regulates the $\alpha \mathrm{v} \beta 6$ expression owing to its vital role in forming heterodimer with $\alpha \mathrm{v}$ proteins. ITGB6 is largely expressed in developing tissues, wound healing process and human epithelial malignancies but not in the normal epithelia. Recent studies showed that ITGB6 plays an important role in multiple human cancers, such as brain cancer, colon cancer and oral cancer, and related to the clinical progression[21-23]. Therefore, ITGB6 might play a role in pancreatic cancer progression.

Our present study revealed that ITGB6 was not or low expressed in normal pancreatic tissue compared with tumor, since ITGB6 was not or low expressed in the normal epithelia pancreatic tissue. Moreover, we performed in-silico TCGA analysis by GEPIA and validated the mRNA expression level of ITGB6 in PAAD showed significantly higher than normal. Further, Cancer specific survival of PAAD was also performed and results showed that high ITGB6 expression was significantly connected with shorter overall survival and disease-free survival in PAAD patients. Our clinical data showed that patients with high ITGB6 expression by immunohistological analysis are more likely to harbor vicious TNM stage, big tumor size and vascular invasion, whose cancer OS time and disease-free survival time were decreased compared with the low expression group. Our results showed that high ITGB6 expression had significantly higher percentage of lymph node metastasis, in accordance with various studies concerning the function of integrin $\beta 6$ in metastasis process[28-30].

Several studies pointed that ITGB6 may promote cancer from the in situ to the invasive stage and from the invasive stage to regional and distant metastasis in different human epithelial malignancies[22,23,31]. Krisha et al. showed that HER2+ breast cancer had elevated levels of ITGB6 and increased regional and distant metastasis which might mediated by the ITGB6 through canonical Rho-Rac pathway[28]. To confirm this clinical finding we observed, we generated ITGB6 targeting shRNA with recombinant lentivirus to silence its expression in pancreatic cancer cells, then validated by western blot assay. We investigated some metastasis-associated proteins expression, such as MMP2, MMP-9, while ITGB6 silencing. Consistent with these results, the mechanism of metastasis suggested that ITGB6 regulated MMP2 and MMP9 to influence the cancer invasive abilities[32]. In breast cancer, Kate el al. demonstrated that using antibody (264RAD) targeting ITGB6 alone or plus trastuzumab might benefit patients with high-risk and trastuzumab-resistant breast cancer[33].In immunotherapy, Lynsey et al. evaluated targeting $\alpha v \beta 6$ chimeric antigen receptor-engineered $\mathrm{T}$ cells for in which aberrant expression of this integrin and developed a therapy in phase 1 for patients with other untreatable $\alpha v \beta 6$-expressing tumors[34]. In addition, our results showed that knockdown ITGB6 greatly reduced proliferation through regulating Ki67 and PCNA proteins in of PANC-1 and BxPC-3 cell lines in vitro. In recent years, 
many biomarkers have been given more attention for diagnosis and treatment for the tumor and non-tumor diseases[35-39]. In summary, the present study demonstrated that silencing ITGB6 in pancreatic cancer cell lines might inhibit the cell behaviors by regulating the concerning proteins. However, the key signaling pathway and molecular mechanism of ITGB6 still needed to be elucidate.

\section{Conclusions}

Taken together, we firstly demonstrated that elevated ITGB6 expression was significantly correlated with poor clinical outcome in pancreatic cancer. Then we studied that functional role of ITGB6 by knockdown in vitro and in vivo showed significantly inhibit cancer cells proliferation and the ability of invasion or migration. Hence, ITGB6 might be a potential target of pancreatic cancer for the future study.

\section{Declarations}

Ethics Approval and consent to participate: All applicable international, national, and/or institutional guidelines for the care and use of human specimens and animals were followed. In our hospital, we have achived the informed consents of patients before the surgerys in the surgical informed consents and agreement. We can not provide these materials because of the Chinese language and privacy protection. The animal study was carried out in accordance with the guidelines approved by the Animal Experimentation Ethics Committee of Academy of Medical Sciences \& Sichuan Provincial People's Hospital. The protocol was approved by the Committee of Academy of Medical Sciences \& Sichuan Provincial People's Hospital, all surgery was performed under sodium pentobarbital anesthesia, and all efforts were made to minimize suffering.

Consent for publication: All of the authors have agreed to publish this article in your journal if it is accepted.

Availability of data and material: The dataset supporting the conclusions of this article is included within the article.

Competing interests: The authors declare that they have no competing interests.

\section{Author Contributions:}

Chao Zhong and Zhi-Xi Li carried out the experiment of molecular biology and drafted the manuscript. Ling-Jing Yang, Gang Wu, and Bo Xiang carried out the animal experiment. Yu-Lan Wang and Qing Zhou participated in the design of the study and performed the statistical analysis. Qing Zhou conceived of the study, and participated in its design and coordination and helped to draft the manuscript. All authors read and approved the final manuscript.

Acknowledgement:This work was supported by the Special Foundation of Sichuan Province People's Hospital,No 2018ZX01. 
Endnotes: None.

\section{Take home message:}

The increased expression of ITGB6 was significantly correlated with poor clinical outcome in both our clinical data and TCGA data of pancreatic cancer. Furthermore, functional assays revealed that knockdown ITGB6 in vivo and in vitro might inhibit cancer cells proliferation and the ability of invasion or migration. Our data suggests that ITGB6 is associated with pancreatic cancer malignant progression. Hence, ITGB6 may be served as a potential target of pancreatic cancer in clinical treatment.

No additional data available.

\section{References:}

1. Rahib L, Smith BD, Aizenberg R, Rosenzweig AB, Fleshman JM, et al. (2014) Projecting cancer incidence and deaths to 2030: the unexpected burden of thyroid, liver, and pancreas cancers in the United States. Cancer Research 74: 2913.

2. Torre LA, Bray F, Siegel RL, Ferlay J, Lortet-Tieulent J, et al. (2015) Global cancer statistics, 2012. CA Cancer J Clin 65: 87-108.

3. Hidalgo M (2010) Pancreatic Cancer. New England Journal of Medicine 362: 1605-1617.

4. Chan A, Diamandis EP, Blasutig IM (2013) Strategies for discovering novel pancreatic cancer biomarkers. Journal of Proteomics 81: 126-134.

5. Yachida S, Jones S, Bozic I, Antal T, Leary R, et al. (2010) Distant metastasis occurs late during the genetic evolution of pancreatic cancer. Nature 467: 1114-1117.

6. Kamisawa T, Wood LD, Itoi T, Takaori K (2016) Pancreatic cancer. Lancet 388: 73-85.

7. Hartwig W, Werner J, Jager D, Debus J, Buchler MW (2013) Improvement of surgical results for pancreatic cancer. Lancet Oncol 14: e476-485.

8. Stark A, Donahue TR, Reber HA, Hines OJ (2016) Pancreatic Cyst Disease: A Review. Jama 315: 1882-1893.

9. Barczyk M, Carracedo S, Gullberg D (2009) Integrins. Cell and Tissue Research 339: 269.

10. Johnson MS, Lu N, Denessiouk K, Heino J, Gullberg D (2009) Integrins during evolution: evolutionary trees and model organisms. Biochim Biophys Acta 1788: 779-789.

11. Ganguly KK, Pal S, Moulik S, Chatterjee A (2013) Integrins and metastasis. Cell Adhesion \& Migration 7: 251-261.

12. Hynes RO (2002) Integrins: bidirectional, allosteric signaling machines. Cell 110: 673.

13. Krissansen GW, Yuan Q, Jenkins D, Jiang WM, Rooke L, et al. (1992) Chromosomal locations of the genes coding for the integrin beta 6 and beta 7 subunits. Immunogenetics 35: 5861.

14. Fernandez-Ruiz E, Sanchez-Madrid F (1994) Regional localization of the human integrin beta 6 gene (ITGB6) to chromosome 2q24-q31. Genomics 21: 638-640.

15. Campbell ID, Humphries MJ (2011) Integrin Structure, Activation, and Interactions. Cold Spring Harbor Perspectives in Biology 3: 594-601.

16. Thomas GJ, Nyström ML, Marshall JF (2006) Alphavbeta6 integrin in wound healing and cancer of the oral cavity. Journal of oral pathology \& medicine : official publication of the International Association of Oral Pathologists and the American Academy of Oral Pathology 35: 1-10.

17. Breuss JM, Gallo J, Delisser HM, Klimanskaya IV, Folkesson HG, et al. (1995) Expression of the beta 6 integrin subunit in development, neoplasia and tissue repair suggests a role in epithelial remodeling. Journal of Cell Science 108 ( Pt 6): 2241-2251.

18. Bandyopadhyay A, Raghavan S (2009) Defining the role of integrin alphavbeta6 in cancer. Current Drug Targets 10: 645-652.

19. Munger JS, Sheppard D (2011) Cross Talk among TGF- $\beta$ Signaling Pathways, Integrins, and the Extracellular Matrix. Cold Spring Harbor Perspectives in Biology 3: a005017.

20. Ghannad F, Nica D, Fulle MI, Grenier D, Putnins EE, et al. (2008) Absence of avß6 Integrin Is Linked to Initiation and Progression of Periodontal Disease. American Journal of 
Pathology 172: 1271.

21. Li HX, Zheng JH, Fan HX, Li HP, Gao ZX, et al. (2013) Expression of alphavbeta6 integrin and collagen fibre in oral squamous cell carcinoma: association with clinical outcomes and prognostic implications. J Oral Pathol Med 42: 547-556.

22. Vogetseder A, Thies S, Ingold B, Roth P, Weller M, et al. (2013) av-Integrin isoform expression in primary human tumors and brain metastases. International Journal of Cancer Journal International Du Cancer 133: 2362-2371.

23. Bates RC, Bellovin DI, Brown C, Maynard E, Wu B, et al. (2005) Transcriptional activation of integrin $\beta 6$ during the epithelial-mesenchymal transition defines a novel prognostic indicator of aggressive colon carcinoma. Journal of Clinical Investigation 115: 339-347.

24. Niu J, Li Z. (2017) The roles of integrin $\alpha v \beta 6$ in cancer. Cancer Lett 403:128-137.

25. Zheng C, Zhou Y, Huang C, Zhang Z, Liu YI, Xu Y. (2015) Establishment of a rat autogenous arteriovenous fistula model following 5/6 nephrectomy. Exp Ther Med 10(1):219-224.

26. Elayadi AN, Samli KN, Prudkin L, Liu YH, Bian A, et al. (2007) A peptide selected by biopanning identifies the integrin alphavbeta6 as a prognostic biomarker for nonsmall cell lung cancer. Cancer Research 67: 5889-5895.

27. Hazelbag S, Kenter GG, Gorter A, Dreef EJ, Koopman LA, et al. (2007) Overexpression of the alpha $\mathrm{v}$ beta 6 integrin in cervical squamous cell carcinoma is a prognostic factor for decreased survival. Journal of Pathology 212: 316.

28. Desai K, Nair MG, Prabhu JS, Vinod A, Korlimarla A, et al. (2016) High expression of integrin $\beta 6$ in association with the Rho-Rac pathway identifies a poor prognostic subgroup within HER2 amplified breast cancers. Cancer Med 5: 2000-2011.

29. Eberlein C, Kendrew J, Mcdaid K, Alfred A, Kang JS, et al. (2013) A human monoclonal antibody 264RAD targeting av $\beta 6$ integrin reduces tumour growth and metastasis, and modulates key biomarkers in vivo. Oncogene 32: 4406-4416.

30. Van Aarsen LA, Leone DR, Ho S, Dolinski BM, Mccoon PE, et al. (2008) Antibody-mediated blockade of integrin alpha $\mathrm{v}$ beta 6 inhibits tumor progression in vivo by a transforming growth factor-beta-regulated mechanism. Cancer Research 68: 561.

31. Ahmed N, Riley C, Rice GE, Quinn MA, Baker MS (2002) Alpha(v)beta(6) integrin-A marker for the malignant potential of epithelial ovarian cancer. Journal of Histochemistry \& Cytochemistry 50: 1371-1380.

32. Morris DG, Huang X, Kaminski N, Wang Y, Shapiro SD, et al. (2003) Loss of integrin avß6mediated TGF- $\beta$ activation causes Mmp12-dependent emphysema. Nature 422: 169-173.

33. Moore KM, Thomas GJ, Duffy SW, Warwick J, Gabe R, et al. (2014) Therapeutic targeting of integrin $\alpha v \beta 6$ in breast cancer. Journal of the National Cancer Institute 106: dju169dju169.

34. Whilding LM, Parentepereira AC, Zabinski T, Davies DM, Petrovic RM, et al. (2017) Targeting of Aberrant $\alpha v \beta 6$ Integrin Expression in Solid Tumors Using Chimeric Antigen Receptor-Engineered T Cells. Molecular Therapy the Journal of the American Society of Gene Therapy 25: 259.

35. Nisic F, Jovanovic N, Mavija M, Alimanovic-Halilovic E, Nisic A, Lepara O, Cemerlic A. (2019) Vitreous concentrations of vascular endothelial growth factor as a potential biomarker for postoperative complications following pars plana vitrectomy. Arch Med Sci 15(2):449-456.

36. Zhang X, Li H, Kou W, Tang K, Zhao D, Zhang J, Zhuang J, Zhao Y, Ji S, Peng W, Xu Y. (2019) Increased plasma microfibrillar-associated protein 4 is associated with atrial fibrillation and more advanced left atrial remodelling. Arch Med Sci 15(3):632-640.

37. Michalska-Kasiczak M, Bielecka-Dabrowa A, von Haehling S, Anker SD, Rysz J, Banach M. (2018) Biomarkers, myocardial fibrosis and co-morbidities in heart failure with preserved ejection fraction: an overview. Arch Med Sci 14(4):890-909.

38. Patoulias DI. Is miRNA-375 a promising biomarker for early detection and monitoring of patients with type 2 diabetes? (2018) Arch Med Sci Atheroscler Dis. 3:e119-e122.

39. Propofol but not sevoflurane decreases circulating levels of sEGFR and sE-selectin after colorectal cancer surgery DOI: 10.5114/amscd.2019.86740 Archives of Medical Science Civilization Diseases - 1 / 2019 
http://www.termedia.pl/Propofol-but-not-sevoflurane-decreases-circulating-levels-of-sEGFR-andsE-selectin-after-colorectal-cancer-surgery,101,37241,0,1.html not found online.

\section{Figure Legends}

Figure 1. Increased expression of ITGB6 connected with poor clinical outcomes in our clinical data.

(A) Low and high IGTB6 expression in patients by immunohistochemistry were shown.

(B) OS and disease-free survival rates with ITGB6 expression in our clinical data: high ITGB6 protein expression level was significantly connected to a shorter cancer OS time and disease-free survival time compared with low expression group (both $P<0.05$ ).

Figure 2. Increased expression of ITGB6 connected with poor clinical outcomes in TCGA data.

(A) Expression level of ITGB6 in cancer and normal tissues (PAAD: Pancreatic Adenocarcinoma; $* P<0.05)$.

(B) OS and disease-free survival rates with ITGB6 expression in TCGA pancreatic adenocarcinoma data: high ITGB6 expression group $(n=89)$ was significantly associated with shorter cancer OS rate and disease-free survival rate compared with low expression group $(\mathrm{n}=89$; $P=0.025$ and $P=0.041$, respectively).

Figure 3. The mRNA and protein expression level of ITGB6 in generated stable cell lines.

The both mRNA and protein expression level of ITGB6 was significantly decreased by (A) RTqPCR and (B) western blotting compared to control cells.

All results are performed at least three independent experiments. $(* P<0.05)$.

Figure 4. Role of ITGB6 on the proliferation in PANC-1 and BxPC-3 cell lines.

(A) Colony formation assay. Results showed that compared to control cells, stable knockdown ITGB6 in PANC-1 and BxPC-3 cells by recombinant lentivirus significantly decreased the ability of proliferation in vitro $(P<0.05)$.

(B) MTT assay. Decreased OD value in ITGB6 silencing group was observed compared to control in vitro $(P<0.05)$.

(C) and (D) Western blotting revealed that Ki67 and PCNA, proliferation related proteins, were significantly decreased in ITGB6 silencing group compared to control (both $P<0.05$ ).

All results are performed at least three independent experiments. $\left({ }^{*} P<0.05\right)$.

Figure 5. Role of ITGB6 on migration and invasion in PANC-1 and BXPC-3 cell lines

(A) Cell scratch assays. Results showed comparing with control, the ITGB6 silencing cells showed bigger scratch space in PANC-1 and BxPC-3 cell lines, respectively $(P<0.05)$. 
(B) Cell invasion assays. The results suggested that the invasive cell numbers in ITGB6 shRNA treated group were significantly less than control $(P<0.05)$.

(C) and (D) Western blotting showed that MMP2 and MMP9, the metastatic associated proteins, were significantly decreased in ITGB6 silencing group compared to control (both $P<0.05$ ).

All results are performed at least three independent experiments. $(* P<0.05)$.

\section{Figure 6. Inhibitory role of ITGB6 silencing in vivo.}

(A) Xenograft assay. After 4 weeks management, the tumor volume of down-regulated ITGB6 group was observably smaller than control $(P<0.05)$.

(B) Western blotting showed that ITGB6 was dramatically reduced by our recombinant lentivirus in injected tumor xenografts in nude mice $(P<0.05)$.

All results are performed at least three independent experiments. $\left({ }^{*} P<0.05\right)$.

Table 1. Relationships of ITGB6 and clinicopathological characteristics in our clinical data of 78 patients with pancreatic adenocarcinoma.

\begin{tabular}{|c|c|c|c|c|c|}
\hline \multirow[b]{2}{*}{ Feature } & \multirow[b]{2}{*}{ All $n=78$} & \multicolumn{2}{|c|}{ ITGB6 expression } & \multirow[t]{2}{*}{$\chi^{2}$} & \multirow[t]{3}{*}{$P$} \\
\hline & & Low & High & & \\
\hline & & $n=28$ & $\mathbf{n}=\mathbf{5 0}$ & & \\
\hline Age (year) & & & & 2.842 & 0.092 \\
\hline$<65$ & 46 & 13 & 33 & & \\
\hline$\geq 65$ & 32 & 15 & 17 & & \\
\hline Gender & & & & 2.457 & 0.117 \\
\hline Male & 48 & 14 & 34 & & \\
\hline Female & 30 & 14 & 16 & & \\
\hline pTNM stage & & & & 4.213 & $0.040 *$ \\
\hline I & 30 & 15 & 15 & & \\
\hline II-III & 48 & 13 & 35 & & \\
\hline Tumor grade & & & & 1.388 & 0.239 \\
\hline Low & 58 & 23 & 35 & & \\
\hline
\end{tabular}


High

Tumor size

$<5$

$\geq 5$

Lymph node

metastasis

Yes

50

28

Vascular

invasion

Yes

No

46

32
5

15
58

20

11

9

17

41
$4.265 \quad 0.039 *$
$0.268 \quad 0.605$

31

19
$4.690 \quad 0.030 *$

34

16 
A

100

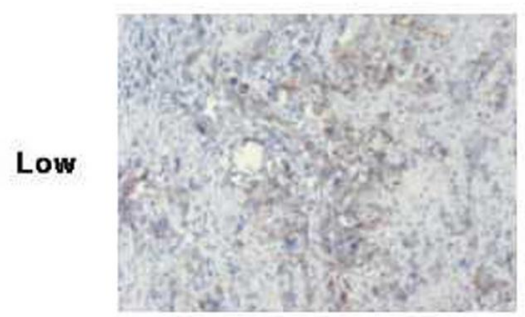

High

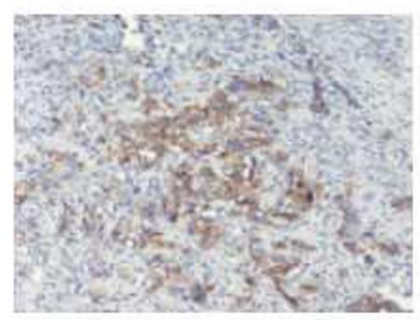

B

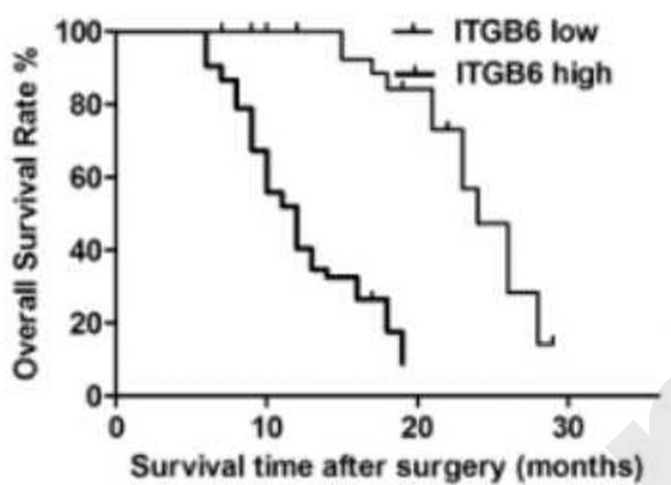

200
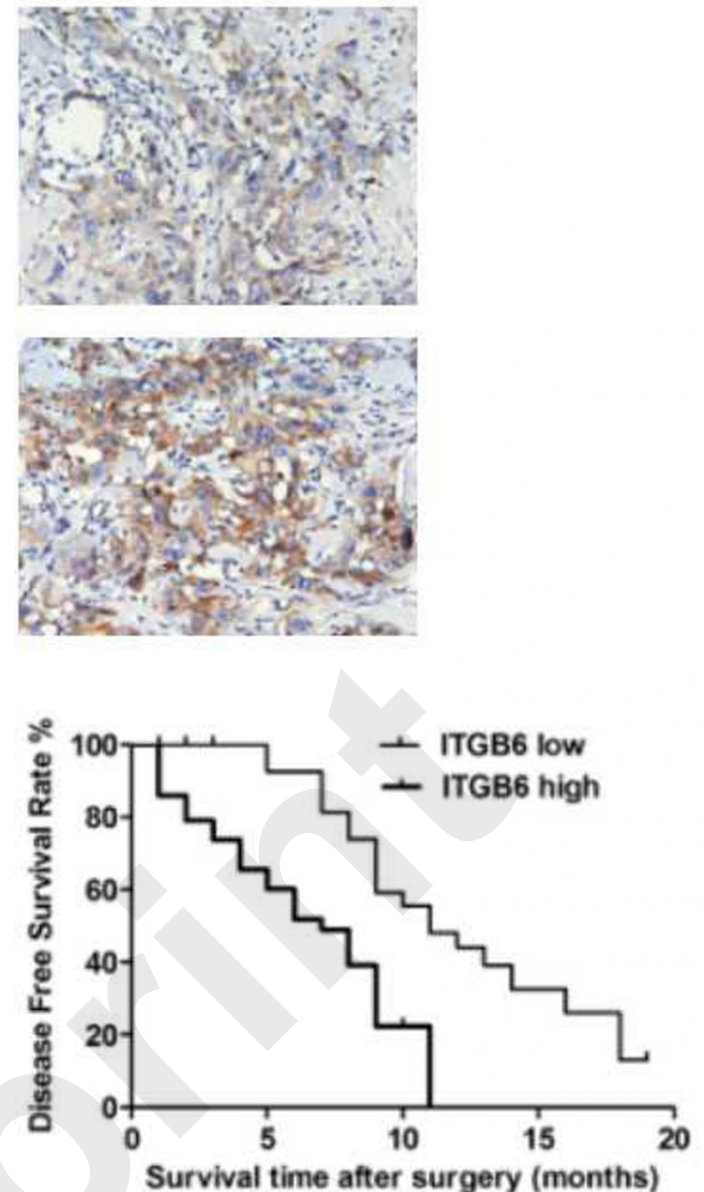
A

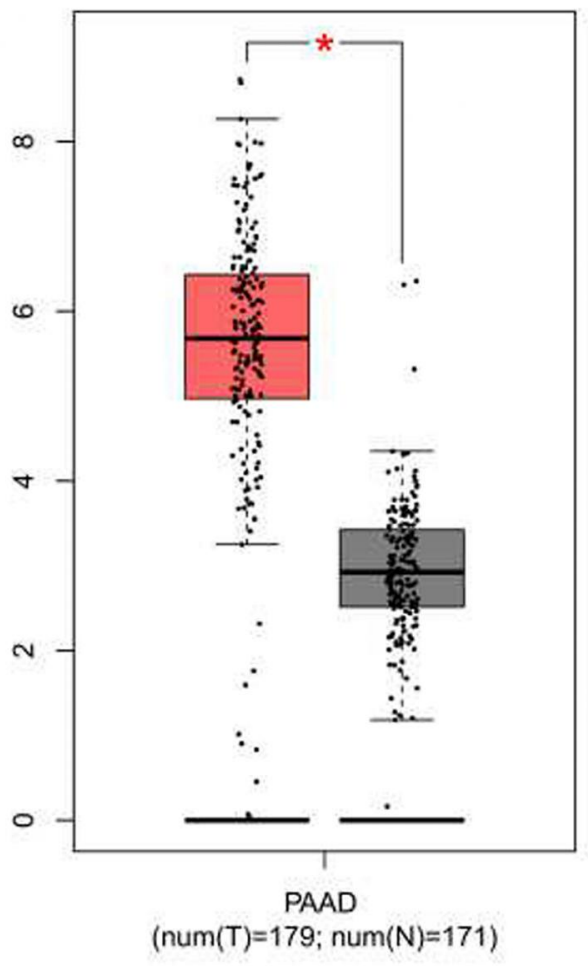

B

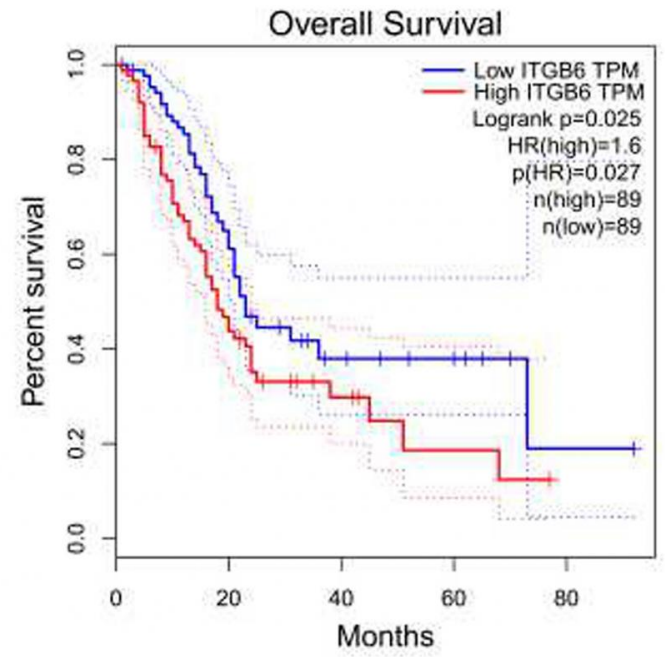

C

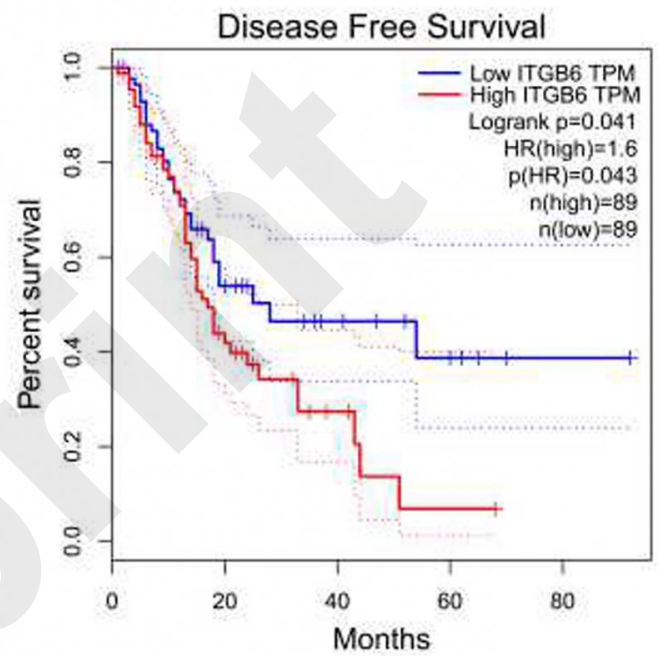


A
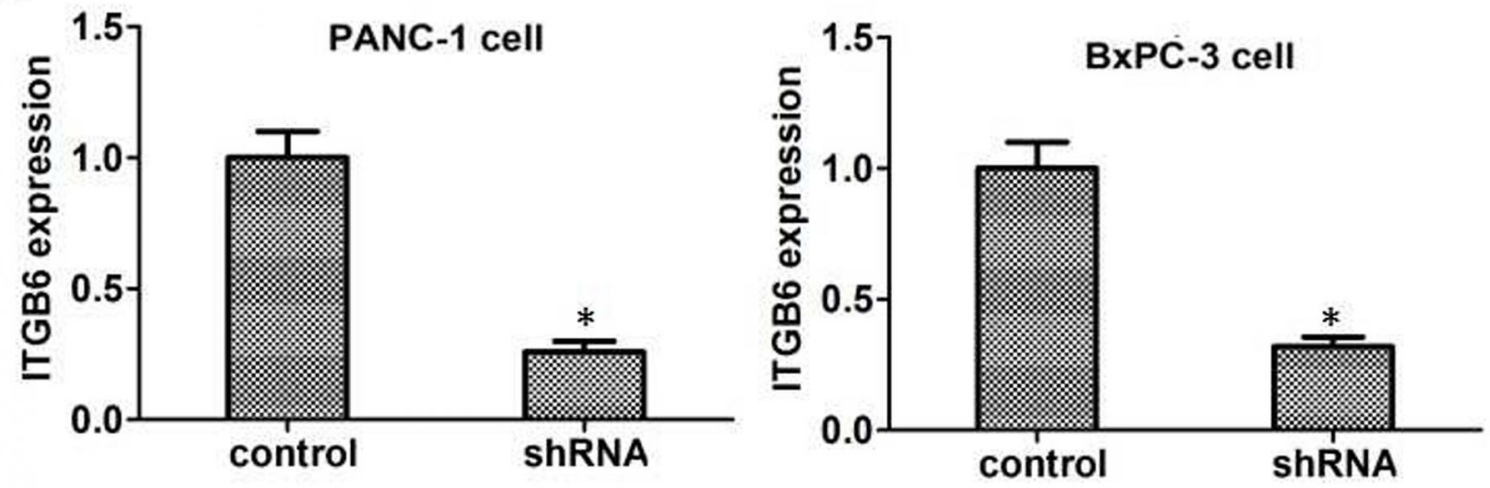

B
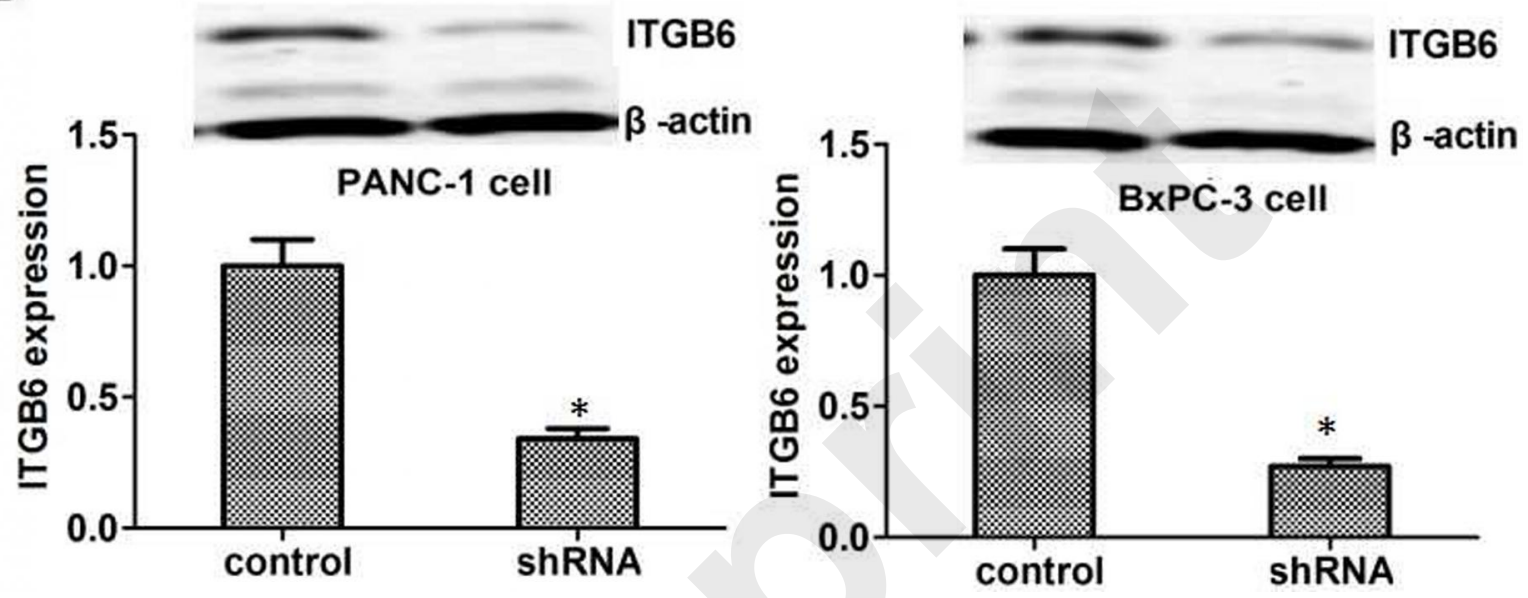
A
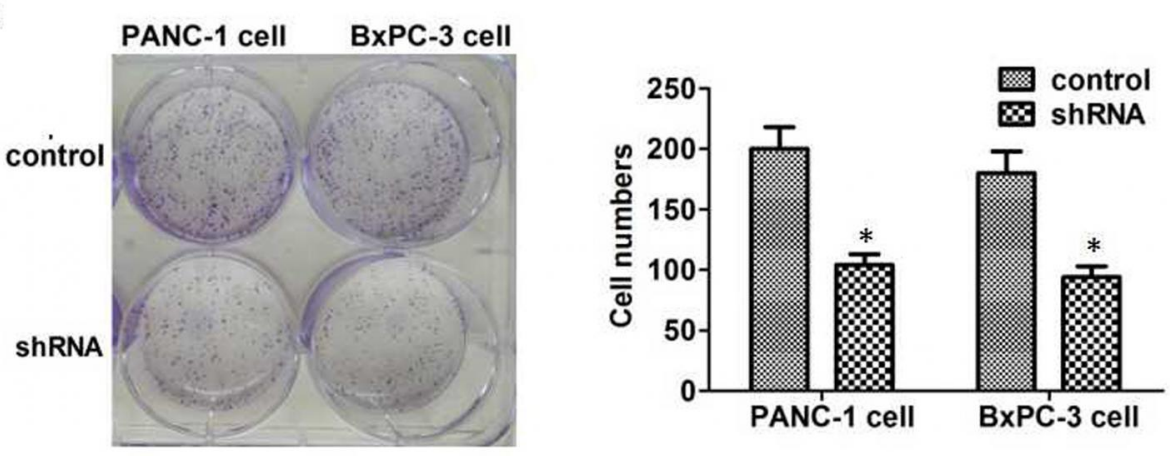

B
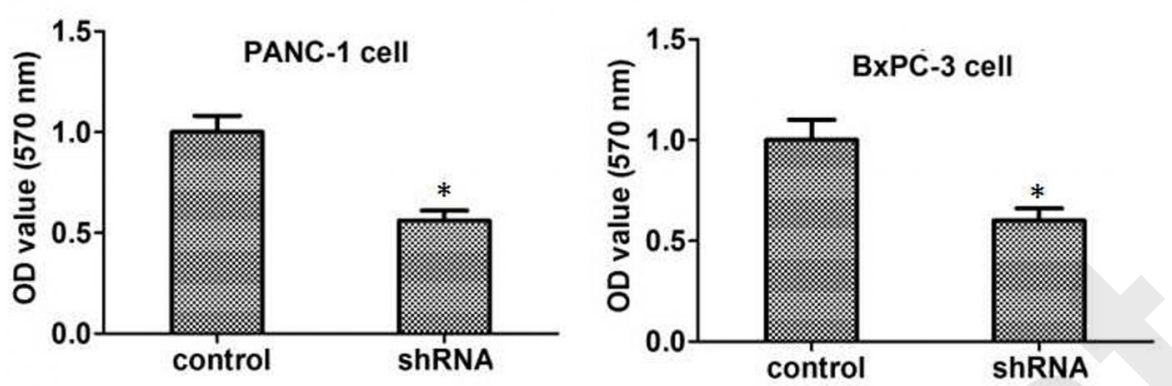

C

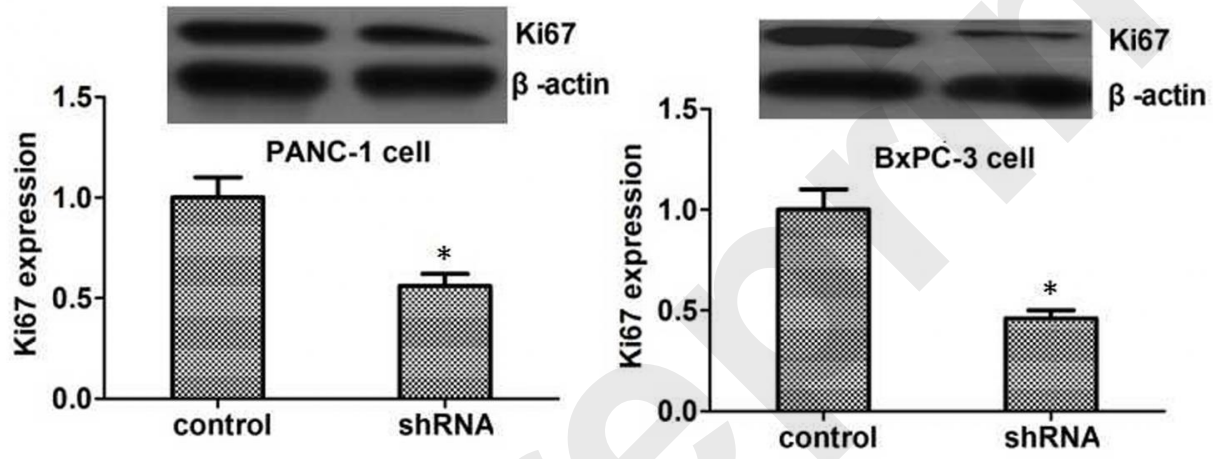

D
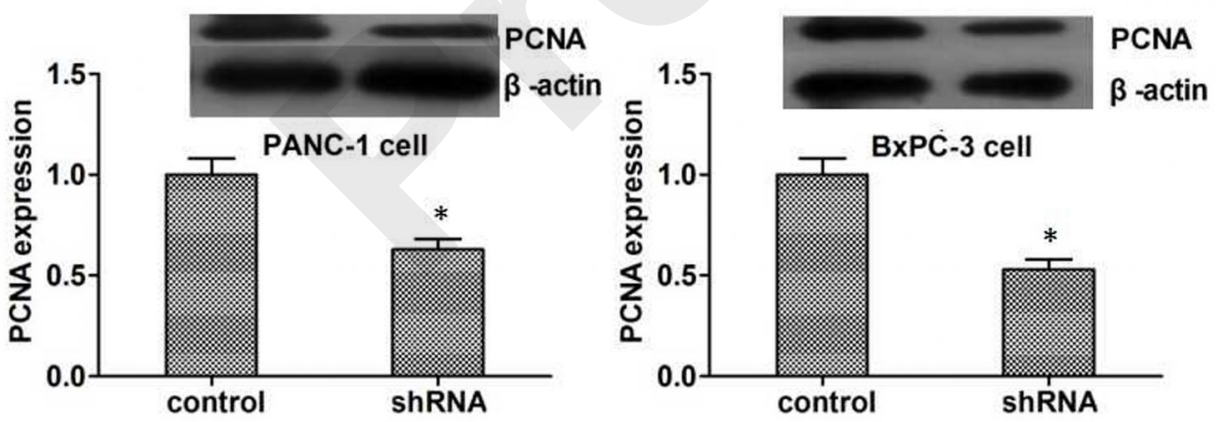
A
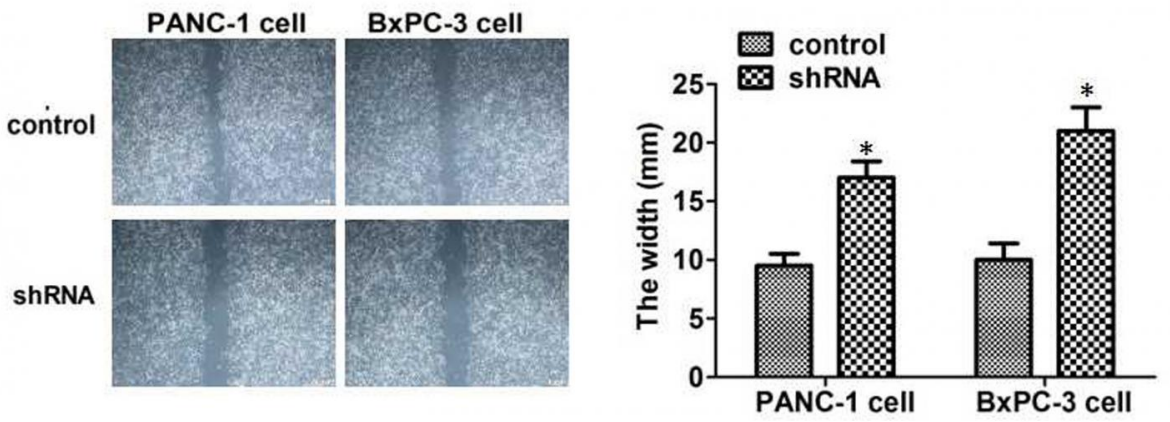

B
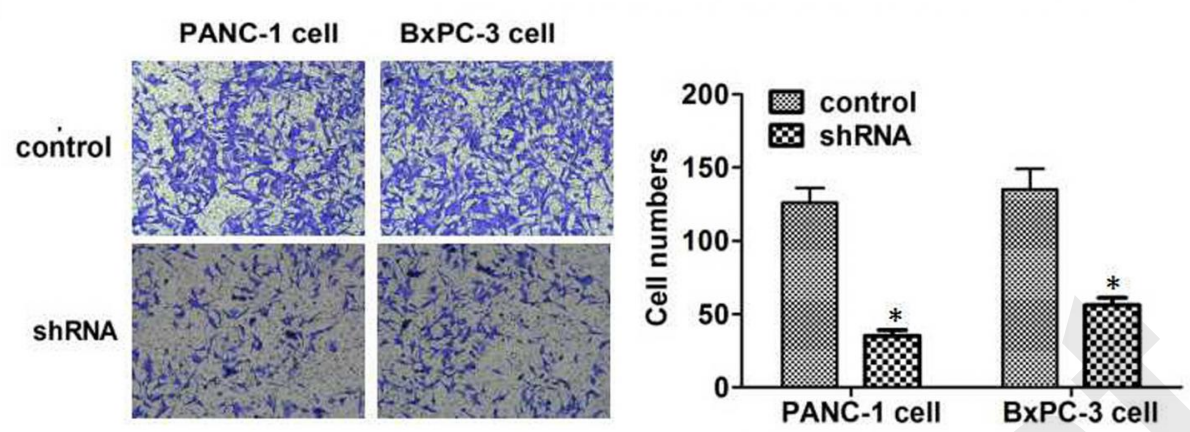

C
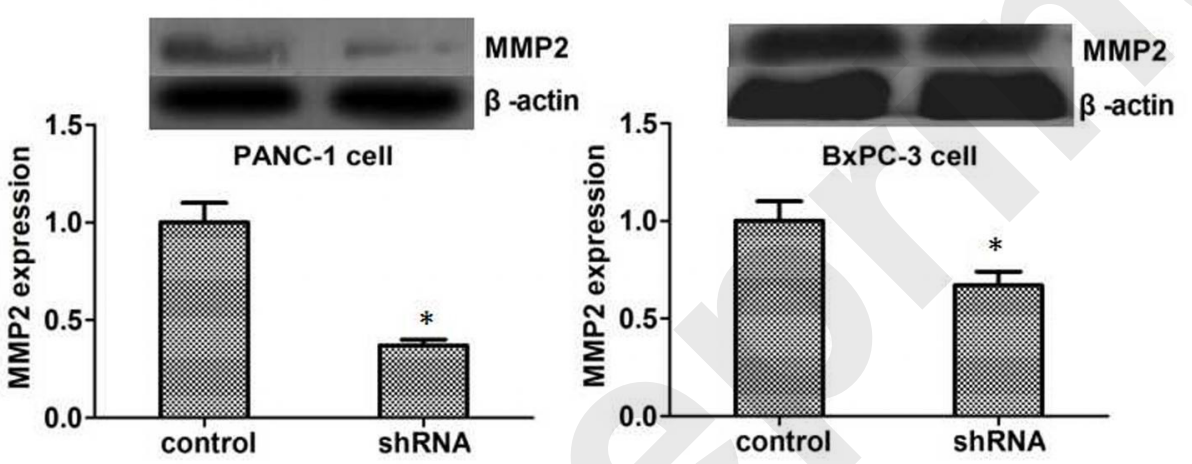

D
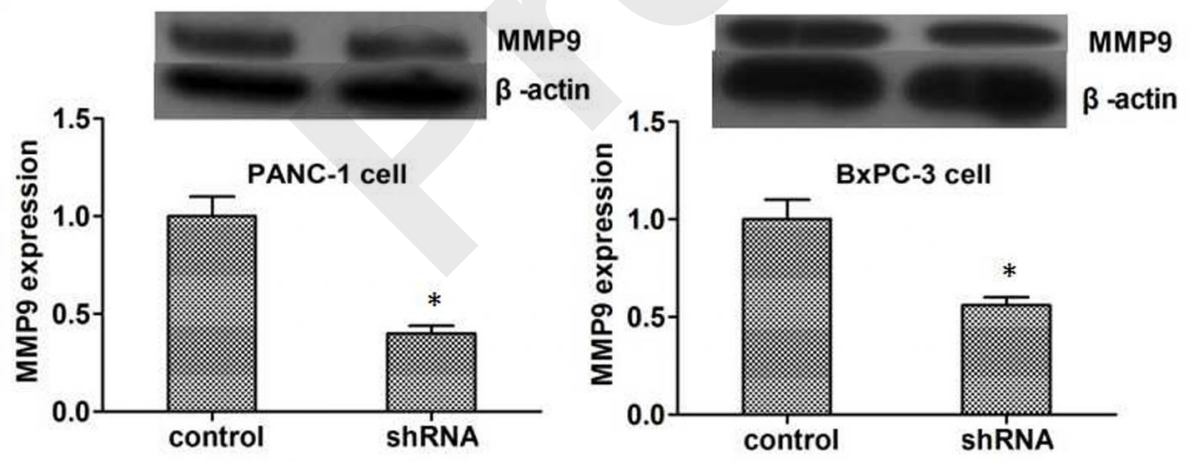
A

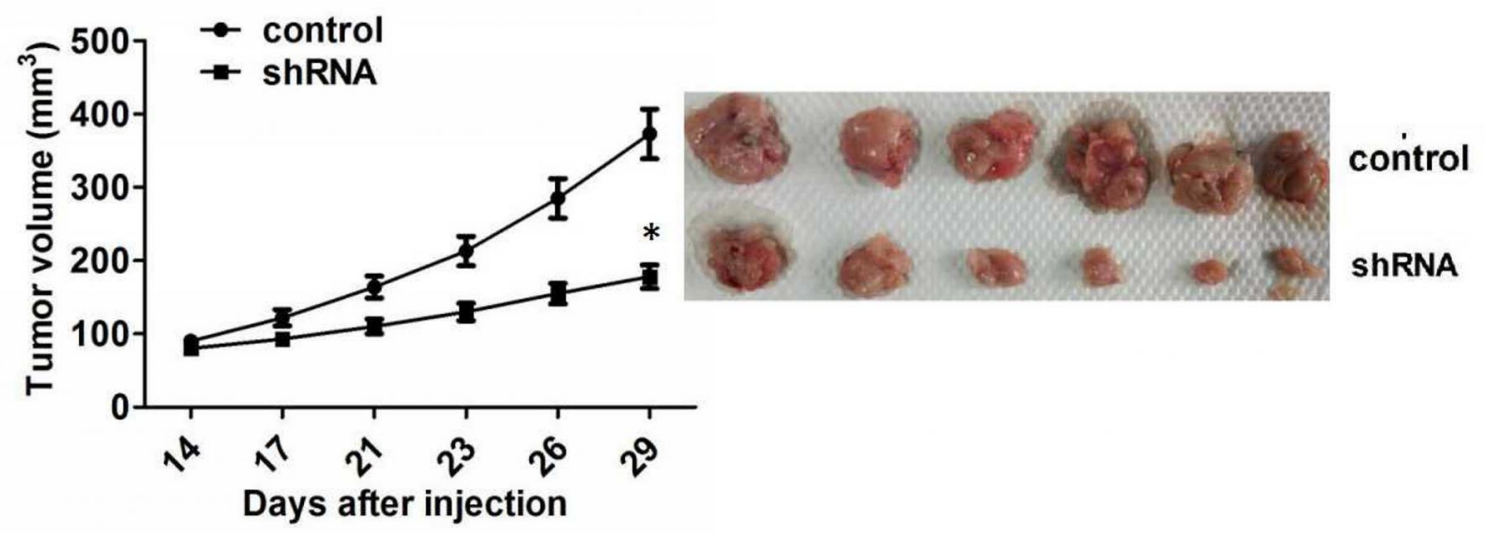

B

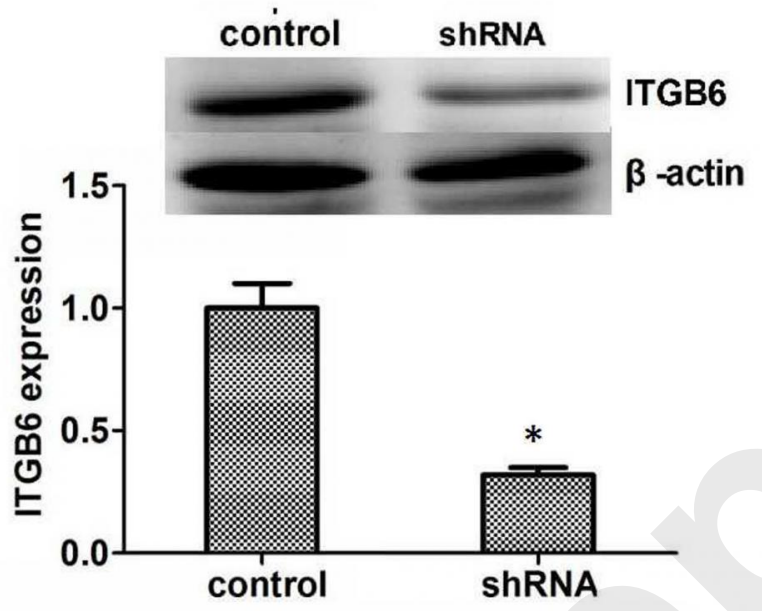

